\title{
Attributes Inequality in Multidimensional Poverty MeasuresFuzzy Modeling
}

Besma Belhadj ( $\square$ besmabelhaj14@gmail.com )

University of Tunis El Manar: Universite de Tunis El Manar https://orcid.org/0000-0001-7464-6208

\section{Research Article}

Keywords: multidimensional poverty, membership function of poor, membership function of inequality, fuzzy set operators, attributes inequality.

Posted Date: January 3rd, 2022

DOl: https://doi.org/10.21203/rs.3.rs-1110060/v1

License: (c) (i) This work is licensed under a Creative Commons Attribution 4.0 International License. Read Full License 


\title{
Attributes Inequality in Multidimensional Poverty Measures Fuzzy Modeling
}

\author{
Besma Belhadj ${ }^{1,2}$ \\ ${ }^{1}$ LaReQuad, FSEGT, University of Tunis ElManar, Tunis Tunisia \\ besmabelhaj14@gmail.com \\ ${ }^{2}$ LIRS, IHE Tunis
}

\begin{abstract}
Poverty is recently considered to be a multidimensional one. That is to say the poor can suffer multiple disadvantages at the same time. This paper aims to further develop and refine the multidimensional poverty measure using Fuzzy Sets Theory (FST). The application of FST starts with the choice of membership functions and the rules to manipulate to integrate attributes inequality in multidimensional poverty measure. An application based on individual well-being data from Tunisian households in 2015 is presented to illustrate use proposed concepts.
\end{abstract}

Keywords: multidimensional poverty; membership function of poor; membership function of inequality, fuzzy set operators, attributes inequality.

JEL classification: P46; I32; D81; C00; 


\section{Introduction}

The poor may have poor health or malnutrition, a lack of clean water or electricity, poor quality of work or little schooling. However, he can suffer multiple disadvantages at the same time. And to capture the reality of poverty it must to focusing on many factors and move to a multidimensional measure of poverty. Multidimensional poverty measure can be used to create a more comprehensive picture. It reveals the range of different disadvantages that the poor experience. As well as providing a headline measure of poverty, multidimensional measure can be broken down to reveal the poverty level in different areas of a country and among different sub-groups of people.

Inequality is about the distances that separate individuals and groups living in a society. Inequality, however, is not a simple concept. Distances can be measured in several different dimensions and as a consequence inequality can refer to rights, capabilities, income, and wellbeing and so on. This suggests that fighting current inequality may help to make social mobility a more concrete phenomenon and reduce a particularly disturbing feature of inequality: its persistence. Too much inequality produces segregation and polarization which put in jeopardy the "cement of society". This implies the much debated disappearance of the middle class which the data in many cases are not able to confirm - raising preoccupations precisely on the ground of its social sustainability. For all these reasons inequality reduction (and more generally, inequality control) should rate high on the political agenda next to poverty. In fact, three I's of poverty (Jenkins and Lambert 1997) are present: It is not only important to reduce the Incidence and Intensity, but also Inequality.

Consideration of Inequality in poverty measurement has been the norm since Sen (1976). Consideration of inequality is natural for measures in cardinal approach - Approaches for Cardinal data (Chakravarty et al.1998; Tsui, 2002; Bourguignon and Chakravarty, 2003, Massoumi and Lugo 2008; Alkire and Foster, 2011, Belhadj, 2016) but not straightforward for measures in counting approach. However, in this last case, inequality can be captured across deprivation counts.

To fine tune a poverty measure to capture inequality, Bossert et al. (2009) uses symmetric or generalized mean across deprivation counts. Also, Jayaraj and Subramanian (2009) and Rippin (2011) use weights deprivation counts by themselves (like FGT). These approaches are merely used for ranking but not suitable for understanding inequality within groups and between groups

To capture inequality in measure poverty, first, we must create a poverty index that is sensitive to inequality. Then, use a separate inequality measure to analyze inequality among the poor and provide more information. An advantage of a separate inequality measure is that if decomposable, it can be used to analyze inequality within groups and between groups.

In this paper, we applied Fuzzy Sets Theory (FST) to propose a separate inequality measure and multidimensional poverty indices sensitive to inequality.

This paper is structured as follows. Section 2 presents a brief description of FST and its application to poverty. Section 3 uses FST to derive alternative separate inequality measure. Section 4 uses FST to derive families of multidimensional poverty indices sensitive to inequality. Section 5 illustrates the use of proposed measures using Tunisian household data of 2015, and Section 6 concludes.

\section{Fuzzy logic, poverty}

Fuzzy logic (Zadeh, 1965) is a form of multi-valued logic derived from FST to deal with approximate reasoning rather than the precise one. 
Let $Y$ be an income distribution, $y_{i}$ the income of the $i^{\text {th }}$ observation unit $(i=1, \ldots, n)$ such as $\left(0 \leq y_{1} \leq \ldots \leq y_{n}\right)$. In fuzzy set theory with fuzzy logic, how a given observation unit can be considered poor or not? We assign, following this approach, to every observation unit a degree of poverty ranging from 0 to 1 according to the corresponding income. This can be done by introducing the so-called membership function, $\mu_{A}\left(y_{i}\right)$, of an observation $i$ to the subset 'poor' , $A$. The set membership values can range (inclusively) between 0 and 1 . Also, the degree of poverty can range between 0 and 1 and is not constrained to the two true values (true (1), false (0)) as in classic predicate logic (Novak et al. 1999). $\mu_{A}\left(y_{i}\right)$ allows us to express to which extent the $i^{\text {th }}$ observation is poor. It quantifies the degree according to which $y_{i}$ belongs to $A$.

In fuzzy logic there are three basic operations on fuzzy sets: union, intersection and complement. Let $\mu_{B}\left(y_{i}\right)$ be membership function of the subset 'very poor', $B$ and $\mu_{A}\left(y_{i}\right)$ be membership function of the subset 'moderately poor', $A$.

The union of fuzzy subsets $A$ and $B$ is a fuzzy set defined by the membership function:

$\mu_{A \cup B}\left(y_{i}\right)=\max \left(\mu_{A}\left(y_{i}\right), \mu_{B}\left(y_{i}\right)\right)$, and the intersection of $A$ and $B$ is $\mu_{A \cap B}\left(y_{i}\right)=\min \left(\mu_{A}\left(y_{i}\right), \mu_{B}\left(y_{i}\right)\right)$.

The complement of $A$ is a fuzzy set defined by the membership function: $\mu_{\bar{A}}\left(y_{i}\right)=1-\mu_{A}\left(y_{i}\right)$.

\section{Fuzzy Lorenz curve, Fuzzy Gini coefficient}

The Gini coefficient, that is usualy defined mathematically based on the Lorenz curve, measures the inequality among values of a frequency distribution (for example, levels of income). A Gini coefficient of zero expresses perfect equality, where all values are the same. A Gini coefficient of one (or 100\%) expresses maximal inequality among values.

Statistics methods used to calculate Gini are based on certain axiomatic Assumptions that who generally ignore the differential convexity of the Lorenz curve. The differential convexity is to extend the convexity concept of function by using a geometrical approach on its epigraph (the set of points on or above the graph of the function). More specifically, since the convexity of a function is equivalent to the convexity of its epigraph, the extension is obtained by substituting a line joining two points in the epigraph by a curve, namely, a solution of a fixed second-order ordinary differential equation.

\subsection{Traditional Gini's index}

The Gini coefficient (Gini, 1912) is based on the Lorenz curve, which plots the proportion of the total income of the population (y axis) that is cumulatively earned by the bottom $x \%$ of the population (Figure 1). The line at 45 degrees thus represents perfect equality of incomes. The Gini coefficient can then be thought of as the ratio of the area that lies between the line of equality and the Lorenz curve ( $A$ in Figure 1$)$ over the total area under the line of equality $(A$ and $B$ in Figure 1); i.e., $I G=A /(A+B)$. 


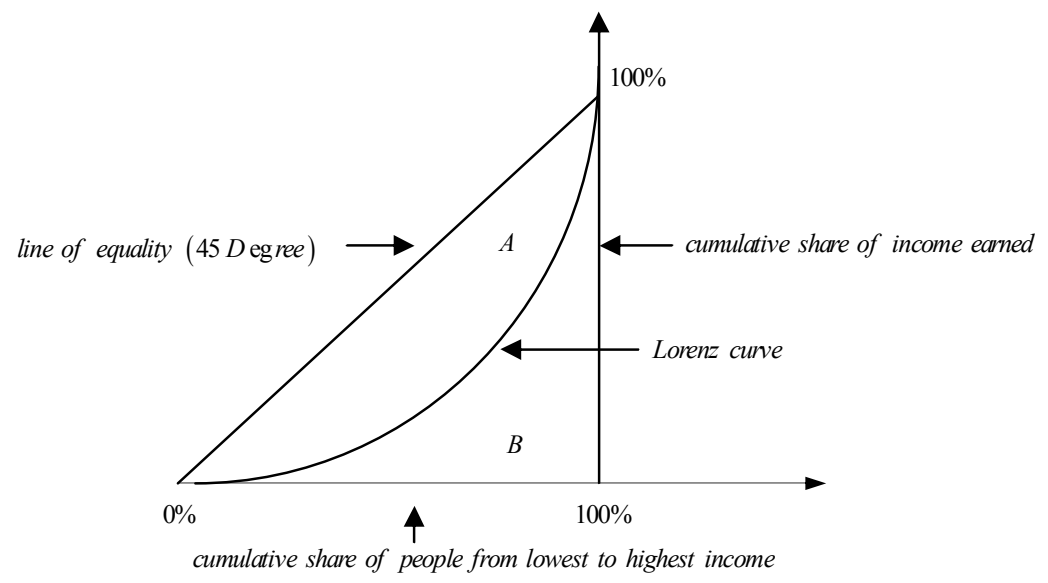

Fig. 1. Lorenz curve

The Gini coefficient can theoretically range from 0 (complete equality) to 1 (complete inequality). It is based on the income levels of individuals and is represented by $I G=1 / 2 n^{2} \bar{w}\left(\sum_{j=1}^{m} \sum_{k=1}^{m} n_{j} n_{k}\left|w_{j}-w_{k}\right|\right), \quad j=1, \ldots ., m$ distinct $\quad$ income $\quad$ groups; $n=\sum_{j=1}^{m} n_{j}$ is the total number of people and $\bar{w}$ is the average/mean of any income $w$ distribution.

The Gini coefficient is also related to the Lorenz curve in a diagrammatic way. The more 'bowed' the Lorenz curve and the higher the degree of income inequality; hence, the higher the Gini.

In the next section, an alternative approach to calculate Gini coefficient is proposed considering the differential convexity of the Lorenz curve. It's about a fuzzy version of the Gini coefficient calculated as agreement index for fuzzy numbers (see Belhadj et al. (2021) for another proposal). Fuzzy version of the Gini coefficient is a measure of inequality. It can be used to indicate how the distribution of income has changed within a country over a period of time, thus it is possible to see if inequality is increasing or decreasing.

\subsection{Agreement Gini's index for fuzzy numbers}

The Lorenz curve is a probability plot comparing the distribution of a variable against a hypothetical uniform distribution of that variable. The Lorenz curve for a probability distribution is a continuous function. However, Lorenz curves representing discontinuous functions can be constructed as the limit of Lorenz curves of probability distributions, the line of perfect inequality being an example.

For the case of discontinuous functions, the imprecision is inherent to the analyst. It is brought by the limits set by the income intervals that "discretize the Lorenz curve" and make it not smooth (the smooth function is locally well approximated as a linear function at each interior point) and does contain break, angle, or cusp.

For a long time, the probability theory has been the predominant theory to model uncertainties of reality. It is based on certain axiomatic assumptions, which are hardly ever tested, when these theories are applied to reality. Fuzzy Theory is one of other theories, which was initially intended to be an extension of classical logic. It uses degrees of truth as a mathematical model of vagueness: 
If $X$ is a collection of objects denoted generically by $x$, then a fuzzy set $T$ in $X$ is a set of ordered pairs: $T=\left\{x, \mu_{T}(x) / x \in X\right\}, 0 \leq \mu_{T}(x) \leq 1$ is called the membership function which maps $X$ to the membership space M.

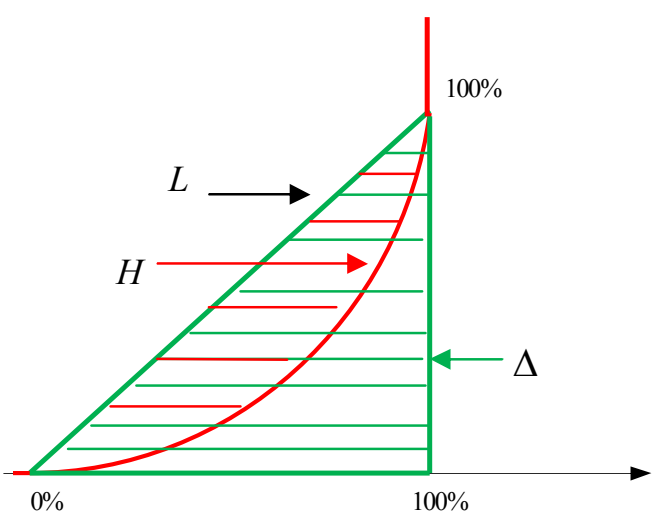

Fig.2. Agreement index for fuzzy numbers

It is a generalization of the indicator function for classical sets. In fuzzy logic, it represents the degree of truth as an extension of valuation. Degrees of truth are often confused with probabilities, although they are conceptually distinct, because fuzzy truth represents membership in vaguely defined sets, not likelihood of some event or condition. Its range is the subset of nonnegative real numbers whose supremum is finite. For $\sup \mu_{T}(x)=1$ : normalized fuzzy set.

Let $\mathrm{S}$ be a vector space or an affine space over the real numbers, or, more generally, over some ordered field. This includes Euclidean spaces, which are affine spaces. Let subsets $H=\left\{\left(y_{j}\right)^{1 / \alpha_{j}}, 0 \leq y_{j} \leq 1, j=1, \ldots, m\right\}$ and $L=\left\{y_{j}, 0 \leq y_{j} \leq 1, j=1, \ldots, m\right\}$ of S. Subsets $H$ and $L$ are convex.

We represent the function $H$ by a fuzzy subset $H \subset R$. Let us now consider a fuzzy number $\Delta \subset R, \Delta$ is a isosceles right triangle, which we call the agreement index of $\Delta$ with regard to $H$ (Kaufmann and Gupta, 1991), the ratio being defined in the following way:

$$
t(\Delta, H)=(\operatorname{area} \Delta \cap H) /(\operatorname{area} \Delta) \in[0,1],
$$

as shown in Figure (2).

Consider the triangle fuzzy number $\Delta \subset R$ shown in Figure (2),

$$
\begin{aligned}
& \forall y_{j} \in R, j=1, \ldots, m \text { groups or attributes : } \\
& \qquad \begin{array}{rlrl}
\mu_{\Delta}\left(y_{j}\right) & =0 & y_{j}<0 \\
& =y_{j} & 0 \leq y_{j}<1 \\
& =1 & y_{j} \geq 1
\end{array}
\end{aligned}
$$

with the following function $H$ : 


$$
\begin{array}{rlrl}
\mu_{H}\left(y_{j}\right) & =0 & y_{j}<0 \\
& \left.\left.=y_{j}^{1 / \alpha_{j}} \in R, \alpha \in\right] 0,1\right]: \quad 0 \leq y_{j}<1 \\
& =1 & y_{j} \geq 1 \\
y_{j} & =\frac{1}{\bar{y}_{j}} \int_{0}^{\alpha_{j}} F^{-1}\left(\bar{y}_{j}\right) d \bar{y}_{j} \\
\bar{y}_{j} & =\int_{0}^{\infty} y_{j} f\left(y_{j}\right) d y_{j}
\end{array}
$$

$\mu_{\Delta}$ and $\mu_{H}$ denote the degrees of truth i.e membership of group $j=1, \ldots ., m$ respectively to subsets $\Delta$ and $H$ (Figure 2), $y_{j}$ is incomes ratio; $F^{-1}($.$) is the quantile function and$ $\alpha_{j}$ is the degree of convexity of the Lorenz curve (Figure 2).

We must now describe the surface $A \cap H$ and compute its area as follows,

$$
\begin{aligned}
& y_{j}=y_{j}^{1 / \alpha_{j}} \text { gives } y_{j}=0 \\
& y_{j}=y_{j}^{1 / \alpha_{j}} \text { gives } y_{j}=1
\end{aligned}
$$

$\forall y_{j} \in R, j=1, \ldots, m$ groups (see appendix for the demonstration of the following equations):

$$
\Delta \cap H=\frac{1}{2}-\int_{0}^{y_{j}} y_{j}^{1 / \alpha_{j}} d y_{j}=\frac{1+\alpha_{j}\left(1-2 y_{j}^{\frac{1}{\alpha_{j}}+1}\right)}{2\left(1+\alpha_{j}\right)}
$$

and $\quad$ Area $\Delta \cap H=\frac{1}{2}-\int_{0}^{1} y_{j}^{1 / \alpha_{j}} d y_{j}=\frac{1}{2}-\frac{1}{1 / \alpha_{j}+1}$

$$
\Delta=\int_{0}^{y_{j}} y_{j} d y_{j}=\frac{1}{2} y_{j}^{2}
$$

and $\quad$ Area $\Delta=\int_{0}^{1} y_{j} d y_{j}=\frac{1}{2}$

Thus we find from (6) and (8) that

$$
F G I_{j}=\frac{\Delta \cap H}{\Delta}=\frac{1+\alpha_{j}\left(1-2 y_{j}^{\frac{1}{\alpha_{j}}+1}\right)}{\left(1+\alpha_{j}\right) y_{j}^{2}}
$$

and from (7) and (9), (1) becomes:

$\forall j=1, \ldots, m$ groups 


$$
t_{j}(\Delta, H)=\frac{\frac{1}{2}-\frac{\alpha_{j}}{\alpha_{j}+1}}{1 / 2}=\frac{1-\alpha_{j}}{1+\alpha_{j}}
$$

$t_{j}(\Delta, H)$ is also the ratio of the area ( $A$ in Figure 1$)$ under the line of equality $(A+B$ in Figure 1). It summarizes the information in a fuzzy Lorenz curve: if $\alpha_{j} \rightarrow 0$ the fuzzy Lorenz curve moves outward, producing greater inequality and then $t(\Delta, H)_{j} \rightarrow 1$ and vice versa if $\alpha_{j} \rightarrow 1$ that show $t(\Delta, H)_{j} \rightarrow 0$. We find from (11) that $t_{j}(\Delta, H)$ is very relative to the curvature of the Lorenz curve. This result is important because it proves that the agreement index of $\Delta$ with regard to $H$ cannot be absolute. $\alpha_{j}$ is then the proportional variation of $F G I_{j}$.

Comparing income distributions among countries may be difficult because benefits systems may be different in different countries. The fuzzy Lorenz curve $\mu_{H}\left(y_{j}\right)$ can account for all of these differences. Indeed, for economies with very different income distributions, the fuzzy Lorenz curve can have different shapes and still yields therefore different $F G I$ coefficients.

It is claimed that the $F G I$ is sensitive to the income of the middle classes and to that of the extremes.

\section{Measuring multidimensional poverty}

Poverty covers both monetary (unidimensional) and non-monetary (multidimensional) aspects. Sen (1992) believes that poverty is not the mere lack of income to meet basic needs, but deprivations in basic human capabilities. An income poverty line, $z$, well captures the monetary aspect of poverty but cannot accurately reflect the non-monetary aspects.

\subsection{Traditional monetary and non-monetary approaches}

The traditional monetary approach to poverty uses income or consumption expenditure $y_{i}$ as an indicator of well-being. This approach start by identifying the poor as those with insufficient income to attain minimum basic needs $z$. In the traditional monetary approach to poverty, the individual poverty function is

$$
S_{i}=\frac{z-y_{i}}{z} 1\left(y_{i} \leq z\right)
$$

Then, this approach aggregates the poor shortfall from the poverty line into a poverty index. Poverty headcount, poverty gap, and severity of poverty are the most common indices used in the literature. All these indices belong to the family of Foster-Greer-Thorbecke (FGT) poverty measures (Foster et al. 1984) given by:

$$
F G T_{\tau}=\frac{1}{n} \sum_{i=1}^{n}\left(S_{i}\right)^{\tau} 1\left(S_{i}>0\right),
$$

where $1($.$) is the indicator function, i=1, \ldots, n$ individual and $\tau$ is a parameter indicating the sensitivity of the index to the distribution among the poor. The higher $\tau$ the more sensitive the index is to the poorest persons in the economy. For $\tau=0$, FGT is the headcount, for $\tau=1$ it is the poverty gap, and for $\tau=2$ it represents the severity of poverty. 
The traditional monetary approach to poverty demands a multidisciplinary analysis. Three main socioeconomic conceptual developments were introduced in the last three decades. The first one is the concept of social exclusion including in particular long term and recurrently unemployed and employed in precarious and unskilled jobs (Lenoir, 1974). The second one was introduced by Sen (1999) is the concepts of functioning, capabilities and entitlement. The UNDP $(1997,1998)$ developed a third concept to the analysis and measurement of poverty. Unlike the social exclusion and Sen's approaches, the UNDP approach was made operational because it ends with a proposed measure of poverty. Its annual Human Development Report publishes two Human Poverty Indexes, one for developing countries and another for industrialized countries includes deprivation in longevity, deprivation in knowledge and deprivation in living standard.

The choice of the set of socioeconomic attributes related to the state of poverty is based on the information available. We select the socioeconomic attributes whose lack of, or partial possession of any of those attributes, contributes to the state of a household poverty. They are represented by the m-order vector $X=\left(X_{1}, \ldots, X_{j}, \ldots, X_{m}\right)$ of attributes. As attributes considered in $X$ we find: income of the household; years of schooling of the household head; age, job-status and gender of the household head; years of schooling of the parent of the household head; occupation of the household head, ownership and typology of the household residence; drinkable water; sanitary (bathroom, shower, sewage) services; and number of senior and handicapped persons in the household.

A multivariate measure of poverty purports to arrive at a poverty index as a function of the $\mathrm{m}$ attributes included in $X$ as follows:

$$
F G T_{\tau}=\frac{1}{n} \sum_{i=1}^{n} \sum_{j=1}^{m} w_{j}\left(S_{i j}\right)^{\tau} 1\left(S_{i j}>0\right)
$$

where,

$$
S_{i j}=\frac{z_{j}-y_{i j}}{z_{j}} 1\left(y_{i j} \leq z_{j}\right)
$$

$z_{j}$ is the insufficient income to attain minimum basic needs by attribute $j, y_{i j}$ is the individual income or consumption expenditure by attribute $j$ and $w_{j}$ is the share of attribute $j$.

The index in (14) measure the relative deprivation and poverty corresponding to each attribute included. It identifies the most important attributes or dimensions that need to be addressed to achieve a structural reduction of poverty.

\subsection{Fuzzy monetary and non-monetary approaches}

This section presents an alternative approach to the derivation of monetary and nonmonetary poverty indices using instruments from FST.

The FST is a rigorous method to operationalize a multivariate analysis of poverty. Cerioli and Zani (1990) applied fuzzy set theory to estimate the poverty in Italy. Dagum et al. (1992), Cheli et al. (1994), Cheli and Lemmi (1995), Martinetti (1994), Belhadj and Limam (2012) and Betti et al. (2017), among others made further contributions and applications.

\subsubsection{Fuzzy monetary poverty}

Let $P$ be the set of poor households defined as follows $P=\left\{i: y_{i} \leq z\right\}$. However, if $i \in P$ and there is an uncertainty in the definition of the poverty line, and thereby of the poor, we may 
specify a membership function which indicates the degree to which a household is considered as poor.

The membership function being continuous differentiable and non-increasing (Figure 1), to be used in the empirical illustration, is proposed as follows:

$$
\mu_{P}\left(y_{i}\right)= \begin{cases}1 & \text { if } y_{i}<y_{1} \\ \left(\frac{z-y_{i}}{z-y_{1}}\right)^{\delta} & \text { if } y_{1} \leq y_{i}<z \\ 0 & \text { if } y_{i} \geq z\end{cases}
$$

where $\delta$ is the concavity of the underlying individual poverty function and is related to the extreme poverty aversion parameter involved in Foster et al. (1984). As noted in Atkinson (2003), it seems reasonable in practice to use the traditional values 1 and 2 for $\delta$. A value of 3 for $\delta$ is sometimes used in the unidimensional context to get a measure which is more sensitive to transfers involving the poorest members of the population.

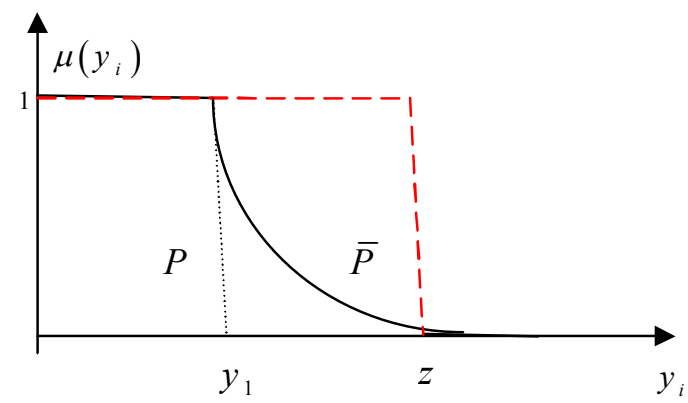

Fig.3. Membership function of poor

If $\delta=0$, we get the standard definition of a crisp set with the membership functions taking a value of 1 for all those with an income below $z$ (graph in red, Figure 3).

The aggregator function across individuals is

$$
U P(Y, z)=\frac{1}{n} \sum_{i=1}^{n}\left[\mu_{P}\left(y_{i}\right) \vee \mu_{\bar{P}}\left(y_{i}\right)\right] 1\left(S_{i}>0\right)
$$

with $\vee$ is the maximal value of $\mu_{P}\left(y_{i}\right)$ and $\mu_{\bar{P}}\left(y_{i}\right)$ because the set of poor still remains $P \cup \bar{P}$,

$$
\bar{P}=\left\{i: z<y_{i} \leq y^{*}\right\} .
$$

This measure complies with extended strong focus, monotonicity, restricted strong monotonicity, restricted continuity, non-decreasingness in poverty domain, Subgroup consistency, anonymity and population invariance. It can be shown that it satisfies the transfer and transfer sensitivity axioms, while being continuous and even decomposable (Zheng. 1997).

\subsubsection{Fuzzy non-monetary poverty}

Let $A_{u, v \ldots}$ be the deprivation space related to the attributes $\{u, v \ldots\}$ and corresponds to the set of points $y_{i} \in R_{+}^{m}$ such that $y_{i j}<z_{j}, \forall j \in\{u, v \ldots\}$. A $k$-deprivation space $A^{k}$ is the subset 
of $R_{+}^{m}$ corresponding to the set of vectors $y_{i}$ such that $y_{i j}<z_{j}$ for at least $k$ attributes (Figure 4), i.e. informal terms $A^{2}=\bigcup_{u \neq v} A_{u, v} \forall\{u, v\} \subseteq\{1, \ldots, m\}$.

By definition, we observe $A^{m} \subset A^{m-1} \ldots \subset A^{1}$. The poverty domain, that is the set of all admissible points in $R_{+}^{m}$ for which individuals deemed poor, is denoted by $\mathrm{P}_{\otimes}$.

The individual muli-attribute poverty function is:

$$
\begin{aligned}
& M P_{i}(Y, z)=\left(\sum_{u, \ldots \ldots=1}^{m} w_{u, v \ldots}\left[\vee \mu_{A_{u, y, .}}\left(y_{i v}\right)\right]^{\beta} 1\left(S_{i u, v \ldots}>0\right)\right)^{\delta / \beta} \\
& \vee \mu_{A_{u, y, .}}\left(y_{i v}\right)=\mu_{A^{m}}=\mu_{\cup_{u \neq v \neq \ldots . .} A_{u, \ldots, \ldots}}, \forall\{u, v \ldots\} \subseteq\{1, \ldots, m\}
\end{aligned}
$$

where $Y$ is an $n \times m$ strictly positive real-valued matrix whose element $y_{i j}$ represents the $i t h$ individual income in the $j t h$ attribute, $w_{u, \ldots}>0$, are attributes' weights. The latter functions are defined as follows:

$$
\mu_{A_{u, v} \ldots}\left(y_{i u, v \ldots}\right)= \begin{cases}1 & \text { if } y_{i u, v \ldots}<y_{1 u, v \ldots} \\ \left(\frac{z_{u, v \ldots}-y_{i u, v \ldots}}{z_{u, v \ldots}-y_{1 u, v \ldots}}\right)^{\delta} & \text { if } y_{1 u, v \ldots} \leq y_{i u, v \ldots}<z_{u, v \ldots} \\ 0 & \text { if } \quad y_{i u, v \ldots} \geq z_{u, v \ldots}\end{cases}
$$

And the aggregator function (across individuals) is given by:

$$
M P(Y, z)=\frac{1}{n} \sum_{i=1}^{n}\left(\sum_{u, v \ldots=1}^{m} w_{u, v \ldots}\left[\vee \mu_{A_{u, v, .}}\left(y_{i v}\right)\right]^{\beta} 1\left(S_{i u, v \ldots}>0\right)\right)^{\delta / \beta}
$$

The parameter $\beta$ in (17) and (19) defines the degree of substitutability between different attributes. When $\beta$ tends to infinity, relative deprivations are non-substitutes and poverty is defined unidimensionally by the attribute deprivation with the highest level of deprivation. But when $\beta=1$, attributes are perfect substitutes and poverty is also defined unidimensionally as a simple weighted sum of attributes. Convexity of attributes, that is concavity in the space of deprivations, requires $\beta \geq 1$.

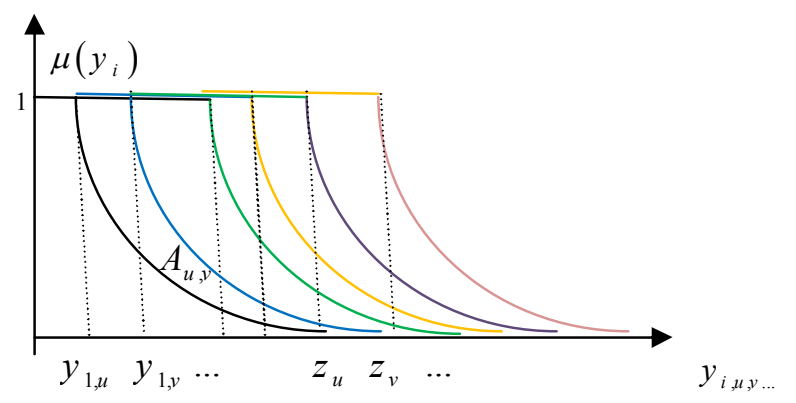

Fig.4. Membership function of attributes 
For the non-monetary poverty it is necessary to deal with situations in which transfers do not alter the marginal distribution of each attribute (Bresson, 2009).

The construction of a measure of non-monetary poverty must then necessarily verify a certain number of properties called axioms (Belhadj and Limam, 2012). We show that our proposition satisfies several axioms in particular attribute decomposability, restricted strong simple transfer, independant transfer and non-ambiguous transfer sensitivity.

\subsubsection{Fuzzy integrated non-monetary poverty}

Poverty is due to inadequate income or education, or poor health, or insecurity, or low selfconfidence, or a sense of powerlessness, or the absence of rights such as freedom of speech (Haughton and Khandker, 2009). However, poverty measures need to be accompanied by inequality measures by attribute to distinguish for example inadequate availability of schools or a corrupt health service. Inequality can be understood at different levels. It occurs between different groups or attributes, either taking into account of their weighting or not (Milanovic, 2012). This different understanding of inequality has different consequences for establishing changes in inequality levels. Inequality refers to discrepancies in income, wealth, education, health, nutrition, space, politics and social identity.

Poverty is related to, yet distinct from, inequality (Haughton and Khandker, 2009). This finding brings us back to integrating a measure of inequality in the measurement of poverty as follows:

$$
\operatorname{IMP}(Y, z)=\frac{1}{n} \sum_{i=1}^{n}\left(\sum_{u, v \ldots=1}^{m} w_{u, v \ldots .}\left[\vee \mu_{A_{u, y,}}\left(y_{i v}\right) \times F G I_{u, v \ldots}\right]^{\beta} 1\left(S_{i u, v \ldots}>0\right)\right)^{\delta / \beta}
$$

FGI is the inequality measure of (10). It concerns with the full distribution of wellbeing. Poverty, $\mu_{A}(y)$, is focused on the lower end of the distribution only - those who fall below a poverty line. The product of the two measures the compound effect (Figure 5).

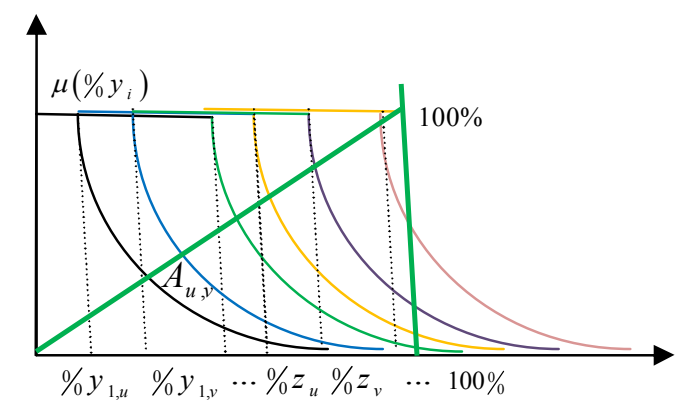

Fig.5. Poverty-Inequality-compound effect by attributes

The measure in (20) is a summary measure of deprivation that includes "poor" and the inequalities that come across economic, education and health/nutrition-related dimensions (attributes). This measure allows showing dimensions or attributes composition and also inequality among poor. It shows joint distribution of deprivation to reach the poorest of the poor. It is Robust to wide range of attributes. 


\section{Inequality in Tunisia}

In this section, we illustrate the use of the proposed inequality measures based on Tunisian household data of 2015 and we make remarks concerning its implementation and practical issues. This exercise highlights the inevitability of making value judgments when analyzing any different income distributions.

\subsection{Inequality by dimension}

Dimensions (socio-economic factors) used here are area and environment. Hence, Tunisia is divided into three parts: The Greater Tunis and two homogenous set namely the Littoral and the Interior. The Greater Tunis area with $25 \%$ of the total population is characterised by very special administrative, social and economic properties. The Tunisian Littoral (Bizerte, Cap-Bon, Sahel, Sfax and Gabes) had known since independence in 1956 an economic and social prosperity. If we compare the per capita expenditure during 2015 we notice that this subdivision is justified. In addition to this regional decomposition, it is necessary to take into account the rural-urban distinction. We also aggregated the rural part of the Greater Tunis and the littoral. Two reasons support this aggregation. First, the size of the rural Greater Tunis is very small, only 167 households and second, the rural of Greater Tunis and those of the rest of the littoral are very similar and can be lumped together to form a homogenous spatial set. This gives five homogenous regions, namely the Greater Tunis, the Urban Littoral, the Urban Interior, the Rural Littoral and Rural Interior.

Table 1 Summary Statistics of the Total Annual

Expenditure Variable (2015)

\begin{tabular}{ll}
\hline \hline First quantile & 2033367 \\
Median & 2962078 \\
Mean & 3791287 \\
Third quantile & 4441689 \\
\hline \hline
\end{tabular}

Data come respectively from the 25144 Tunisian household survey conducted by the Tunisian Institute of Statistics (2015). The survey provides information about total annual expenditures in dinars per household as well as the size of each household. It also provides information about additional indicators of privation, namely economic area and region. A brief summary the total annual expenditure variable is given in Table 1.

Table 2 Fuzzy inequality by area

\begin{tabular}{lc}
\hline \hline & $F G I$ \\
\hline area & 2015 \\
\hline Greater Tunis & 0.64 \\
Urban Littoral & 0.58 \\
Urban Interior & 0.71 \\
Rural Littoral & 0.73 \\
Rural Interior & 0.74 \\
\hline
\end{tabular}

According to Table (2), Rural Interior and Rural Littoral regions have the highest income Gini index in 2015. The remaining regional averages were: Interior Interior, Greater Tunis and Urban Littoral. Using the same method, the rural environnement have the highest income Gini index score in 2015 . 
Table 3 Fuzzy inequality by environment

\begin{tabular}{ll}
\hline \hline & $F G I$ \\
\hline environment & 2015 \\
\hline Urban & 0.51 \\
Rural & 0.62 \\
\hline
\end{tabular}

We highlight that the distribution of income is important for making progress towards the income poverty and the headcount, and when considering the depth of poverty. Indeed, poverty and inequality although theoretically distinct concepts (Atkinson, 1987) are very closely linked as they summarise different aspects of the same. Inequality considers the entire spread of a distribution whereas poverty mainly focus on the lower part of the distribution and is mainly concerned with identifying the poor and summarising this into an indicator that show levels of poverty in a society (Foster et al., 2013).

\subsection{Inequality by attribute, Poverty}

We use the attributes available in the survey which are: expenditures on food and beverages at home and outside home (Alimentation), expenditures of housing including interest on rental or mortgages payments and other expenditures related to periodic maintenance of property (Shelter), expenditures on medicines, health insurance coverage and medical service (Health care), expenditures related to the purchase, maintenance and repair of vehicles as well as public transportation and other related expenses (Transportation), expenditures corresponding to the purchase and phone communication as well as to internet connection and other related expenses (Telecommunication), expenses relative to the payment of fees and the purchase of books and equipments related to public and private schools or universities (Education).

Table 4 Summary Statistics of the Total Annual

Expenditure Variable by attributes (2015)

\begin{tabular}{lllllll}
\hline \hline & Education & Telecom & Transport & Health & Shelter & Foods \\
\hline First quantile & 16 & 54 & 0 & 80 & 507 & 555 \\
Median & 130 & 133 & 93 & 176 & 753 & 789 \\
Mean & 61 & 201 & 279 & 321 & 1103 & 977 \\
Third quantile & 626 & 270 & 267 & 365 & 1224 & 1203 \\
\hline
\end{tabular}

Using a poverty line per day and per individual equal to $\$ 1.90$ (World Bank, 2015), we calculate a threshold per attribute proportional to the share of each attribute (Table 5, column 4). Inequality and poverty by attribute respectively appear in columns (2) and (3) of Table (5).

Table 5 Fuzzy integrated non-monetary poverty

\begin{tabular}{lcccc}
\hline \hline & Attribute Weight & $\begin{array}{c}\text { Attribute } \\
\text { inequality }\end{array}$ & $\begin{array}{c}\text { Attribute } \\
\text { poverty }\end{array}$ & $\begin{array}{c}\text { Attribute poverty line per day } \\
\text { and per individual in \$ }\end{array}$ \\
\hline Education & 0.02 & 0.64 & 0.181 & 0.038 \\
Telecom & 0.07 & 0.46 & 0.123 & 0.133 \\
Transport & 0.09 & 0.71 & 0.155 & 0.171 \\
Health & 0.11 & 0.84 & 0.172 & 0.209 \\
Shelter & 0.37 & 0.75 & 0.145 & 0.703 \\
Foods & 0.34 & 0.82 & 0.211 & 0.646 \\
FGI & & 0.565 & & \\
IMP & & & 0.152 & \\
\hline
\end{tabular}


$I M P=0.152$ (Table 5, end column 3) is the percentage of deprivations poor people experience, as a share of the possible deprivations that would be experienced if all people were deprived in all dimensions.

The poverty headcount ratio tells us which people are poor. IMP also shows how people are poor. But $I M P$ can be unfolded and folded in different ways. So you see how it's made and how it can be changed. For example, to see the contribution of each attribute, we multiply the censored poverty measure by the weight of that attribute.

The multidimensional inequality (Table 5, end column 2) is equal to 0.565 . Marginal distributions of attributes (Table 5, column 2) can shows a situation where two persons switch achievements either one for a single dimension in which both are deprived or for two substitutable dimensions. The resulting transfer specifies conditions under which an alternative form of progressive transfer among the poor that preserves the marginal distributions of attributes and lowers inequality should lower poverty, or at least not raise it.

The dimensional transfer is verified by the class of measures IMP that respect dimensional breakdown and to supplement these with associated inequality measures.

From the results of table (5), we find that the measure IMP in (20) can be used for additional purposes. Beyond measuring poverty and wellbeing, this measure can be adapted to target services and conditional cash transfers or to monitor the performance of programmes. However, the more policy-relevant information there is available on poverty, the better-equipped policymakers will be to reduce it. For example, individuals or households that are deprived in education require a different poverty reduction strategy from that are deprived in housing conditions.

We can deduce that an efficient targeting must depend on whether it is unidimensional or multidimensional poverty that policy is intended to reduce because the value of targeting prescriptions depends on the structure of the transfers.

Indeed, the appropriate indicator to use to design efficient targeting schemes is not the poverty measure themselves. It is the degree of deprivation for each attribute that should be used to identify which group it is most efficient to target. This makes it necessary inter alia to consider important trade-offs between the effect of targeting on the poorest of the poor and the effect of targeting on the speed of income increase among the not-so-poor.

\section{Concluding remarks}

In this paper we interests to measure non-monetary poverty introducing an inequality measure. In this non-monetary poverty measure we introduce population poverty-inequality ratios by attributes and determine their contributions to the total poverty ratio. These ratios provide basic information about the causes of poverty and inequality. They are of paramount importance to the design and implementation of a structural socioeconomic policy to abate the causes of poverty. Hence, they purport to break the mechanism responsible for its intergenerational transmission. This policy, being structural, not cyclical, should aim at a process of structural change with the scope of generating stable, efficient and equitable socioeconomic processes.

An application using individual well-being data from Tunisian households in 2015 are presented to illustrate proposed concepts. Results show that the choice of poverty measures that policy is intended to reduce helps in efficient targeting. The fuzziness involved in choosing one specific poverty index and one specific poverty frontier and the possible sensitivity of targeting prescriptions to that choice make it desirable to use targeting membership function tools. These tools also help assess the normative strength of targeting prescriptions. 


\section{Statements \& Declarations}

\section{Funding}

The author declares that no funds, grants, or other support were received during the preparation of this manuscript.

\section{Competing Interests}

The authors have no relevant financial or non-financial interests to disclose.

\section{Author Contributions}

The author contributed to the study conception and design. The author read and approved the final manuscript.

\section{References}

Atkinson, A., 2003. Multidimensional Deprivation: Contrasting Social Welfare and Counting Approaches. Journal of Economic Inequality 1, 51-65.

Atkinson, A., Bourguignon, F., 1982. The Comparison of Multi-Dimensioned Distributions of Economic Status. Review of Economic Studies 49, 183-201.

Belhadj, B., Limam, M., 2012. Unidimensional and multidimensional fuzzy poverty measures: New approach, Economic Modeling, Vol. 29(4), pp 995-1002.

Belhadj, B., 2016. Inequality among the poor in Poverty Measure Case of Tunisia (2005-2010). OPSEARCH, Vol. 53(2), 409-425.

Belhadj, B., Kaabi, F., Bouanani, M., 2021. A Fuzzy version of Gini Index, Social Indicators Research, Vol.157 (3), pp 1079-1087.

Betti, G., Cheli, B., Lemmi, A., Verma, V., 2006. Multidimensional and Longitudinal Poverty: an Integrated Fuzzy Approach, Fuzzy Set Approach to Multidimensional Poverty Measurement, Economic Studies in Inequality, Social Exclusion and Well-Being, Volume 3, pp. 115-137.

Betti, G., Verma, V., 2008. Fuzzy measures of the incidence of relative poverty and deprivation: a multidimensional perspective. Statistical Methods \& Applications, 17 (2), 225-250.

Betti, G., Gagliardi, F., Verma, V., 2017. Simplified Jackknife Variance Estimates for Fuzzy Measures of Multidimensional Poverty. International Statistical Review, 86 (1), 68-86.

Bourguignon, F., Chakravarty, S., 2003. The measurement of multidimensional poverty. Journal of Economic Inequality 1, 25-49.

Bresson, F., 2009. Multidimensional Poverty Measurement with the Weak Focus Axiom, Document de Travail.

Cerioli, A., Zani, S.,, 1990. A Fuzzy Approach to the Measurement of poverty. In C.Dagum and M.Zenga, editors, Income and Wealth Distribution, Inequality and Poverty, Studies in Contemporary Economics. Spinger Verlag, Berlin, pp. 272-284.

Cheli, B., Ghellini A., Lemmi A., Pannuzi N., 1994. Measuring Poverty in the Countries in Transition, Via TFR Method: The Case of Poland in 1990-1991. Statistics in Transition, 1 (5), 585-636.

Cheli, B., Lemmi, A., 1995. Totally fuzzy and relative approach to the multidimensional analysis of poverty. Economic Notes, 24, 115-134.

Chiappero Martinetti, E. 1994. A New Approach to Evaluation of Well-Being and Poverty by Fuzzy Set Theory. Giornale degli economisti e annali di economia, 53, 367-388.

Chiappero Martinetti, E., Roche, J. M., 2009. Operationalization of the capability approach, from theory to practice: a review of techniques and empirical applications, Contribution to "Debating Global Society: reach and limits of the CA", Fondazione Feltrinelli Part II-Ch1.

Dagum, C., Gambassi, R., Lemmi, A. 1992. New Approaches to the Measurement of Poverty. In Poverty Measurement of Economics in Transition, Polish statistical Association \& Central Statistical office, Warsaw.

Dasgupta, P., Sen, A., Starett, D., 1973. Notes on the Measurement of Inequality. Journal of Economic Theory $6,180-187$.

Deaton, A., Muellbauer J., 1980. An Almost Ideal Demand System. American Economic Review 70, $312-$ 326. 
Efron, B., Tibshirani, R. J., 1993. An introduction to the bootstrap. New York: Chapman and Hall.

Foster, J., Greer, J., Thorbecke, F., 1984. A class of decomposable poverty measures. Econometrica 52, 761-765.

Filippone, A., Cheli, B., D'Agostino, A., 2001. Addressing the Interpretation and the Aggregation Problems in Totally Fuzzy and Relative Poverty Measures, Working Papers of the Institute for Social and Economic Research, paper 2001-22, University of Essex.

Hall, P., 1992. The Bootstrap and Edgeworth Expansion, Springer, New York.

Hayek, F.A., 1960. The Constitution of Liberty, Chicago, University of Chicago.

Haughton, J., Khandker, S. R., 2009. Handbook on poverty and inequality. Washington, DC: World Bank.

INS, 1990. Enquête sur le budget et la consommation des ménages en Tunisie. Ministère du plan, Tunis.

Kakwani, N., 1980. On a class of poverty measures. Econometrica 48, 437-446.

Kaufmann, A., Gupta, M.M., 1991. Introduction to Fuzzy Arithmetic, International Thomson Computer Press.

Milanovic, B., 2012. Global income inequality by the numbers: In history and now (Policy Research Working Paper 6259). Washington, DC: World Bank.

Mills, A.M., Zandvakili, S., 1997. Statistical inference via bootstrapping for measures of inequality. Journal of Applied Econometrics 12, 133-150.

Novák, V., Perfilieva, I., Močkoř, J., 1990. Mathematical principles of fuzzy logic Dodrecht: Kluwer Academic.

Qizilbash, M. 2001. Vague Language and Precise measurement: The case of Poverty; Working Paper 2001-5, School of Economics and Social Studies, University of East Anglia, Norwich.

Ravallion, M., 1994. Poverty Comparisons. Fundamentals of Pure and Applied Economics Series, Harwood Academic Press, Newyork.

Ravallion, M., Bidani, B., 1994. How Robust is a Poverty Profile? The word Bank Economic Review.

Rodgers, J.R., Rodgers, J.L., 2000. Poverty Intensity in Australia. Australian Economic Review, 33(3), 235-244.

Sen, K., 1976. Poverty: An Ordinal Approach to Measurement. Economica 44, 219-231

Sen, K., (1999), Commodities and Capabilities, Oxford University Press, New Delhi.

Shao, J., Tu, D., 1995. The Jacknife and the Bootstrap, Springer, New York

Silber, J., 2011. Multidimensional approaches to poverty measurement: an empirical analysis of poverty in Belgium, France, Germany, Italy and Spain, based on the European panel. Applied Economics, 43 (8), 951-961.

Tsui, K. Y., 2002. Multidimensional Poverty Indices. Social Choice and Welfare 19, 69-93.

United Nation Development Program (1997), Human Development Report, Oxford University Press, New York and Oxford.

United Nation Development Program (1998), Human Development Report, Oxford University Press, New York and Oxford.

Wilkinson, R.H., 1963. A method of generating functions of several variables using analog diode logic. IEEE Transactions on Electronic Computers EC12, 112-129

Zadeh, L., 1965. Probability Theory and Fuzzy Logic are Complementary rather than Competitive. Technometrics 37, 271-276

Zheng, B., 1997. Aggregate Poverty Measures. Journal of Economic Surveys, 11(2), 123-162. 